\title{
Predictor-Corrector Procedures for Analysis of Laminated Plates using Standard Mindlin Finite Element Models
}

\author{
K.Y.Sze*, L.-W.He ${ }^{\#}$ \\ Department of Mechanical Engineering, The University of Hong Kong \\ Pokfulam Road, Hong Kong, P.R.CHINA \\ and \\ Y.K.Cheung \\ Department of Civil Engineering, The University of Hong Kong \\ Pokfulam Road, Hong Kong, P.R.CHINA
}

\begin{abstract}
This paper introduces predictor-corrector methods for finite element analysis of laminated composite plates. The predictor stress, strain and deflection are directly computed from Mindlin finite element models without using shear correction factors. The corrector transverse shear stress is determined from the homogeneous stress equilibrium condition and the predictor in-plane stress by least-square fit. Shear correction factor for each element is estimated as the ratio of the transverse shear energies computed by the predictor transverse shear strain and the corrector transverse shear stress. The corrector deflection is calculated by incorporating the shear correction factor. First, second and third order Mindlin nine-node plate elements are employed for testing the proposed procedure. Its accuracy is demonstrated by a large number of examples.
\end{abstract}

Published in Composite Structures 50: 171-182 (2000).

* Corresponding author. Fax : (852) 2858 5415. Email : kysze@hkukcc.hku.hk

\# on leave from Department of Mechanics, Lanzhou University, Lanzhou, Gansu 730000, P.R.CHINA 


\section{INTRODUCTION}

Considerable research efforts have been reported on development of analytical and numerical models for structural/stress analysis of plates/shells. It has been well-known that the thin plate theory which ignores the shear deformation is inadequate for thick and moderately thick plates/shells. Consequently, the Mindlin theory or the first order Mindlin theory that allows for a constant transverse shear strain along the thickness direction was proposed [1,2]. From equilibrium point of view, the transverse shear stress should vanish at the top and bottom surfaces of the plate. Consequently, a shear correction factor is required to maintain a proper transverse shear stiffness in energy-based numerical methods. The factor as for homogeneous materials can be discarded if higher order Mindlin theories are employed that carry more terms in the series expansion of the inplane displacement in the thickness direction and allow the zero shear traction condition to be satisfied.

A large volume of works in the laminate analysis using higher order Mindlin theories or their variants in which the displacement assumption is independent of the lamination have been reported, see [3-6] for examples. It is clear that the accuracy of the theories increase with their orders. However, they fail in predicting the transverse shear stress due to the $C^{1}$-nature of the assumed inplane displacement in the thickness direction that renders the transverse shear strain continuous. With different elastic moduli in different plies, the predicted transverse shear stress becomes discontinuous and, thus, violates the traction reciprocity at the ply-interface. Another approach in the laminate analysis is the layerwise method in which a piecewise continuous displacement field is assumed in each ply. Its obvious drawback is that a problem can quickly become intractable when the number of plies increases [7-11]. While the in-plane stress prediction is far more accurate than that transverse shear stress prediction in both approaches, a common practice for obtaining a more accurate transverse shear stress is to integrate the derivative of the in-plane stress with respect to the thickness coordinate according to the stress equilibrium condition [4-6,9,10]. It is also possible to start the displacement interpolation with the layerwise approach and condense some of the displacement parameters by enforcing the zero shear traction and traction reciprocity conditions. Provided that the layerwise displacement does not contain excessive number of thickness terms, the net number of displacement parameters can remain constant and does not grow with the number of plies [12-16].

Owing to its versatility, finite element method has been the dominating engineering tool for structural/stress analysis. Among the ad hoc formulated finite element models for laminate analysis, most of them suffer from an increasing number of nodal parameters with the number of plies and/or non-conventional nodal kinetic d.o.f.s which render them obscure to ordinary finite element 
practitioners $[5,7,9-11,14,16]$.

In this paper, simple yet not excessively expensive predictor-corrector procedures for finite element analysis of laminated plates are devised. The finite element solution that includes the predictor displacement, predictor strain and predictor stress is first obtained by using the conventional Mindlin plate finite element models. The corrector transverse shear stress is computed by least square fitting a piecewise continuous shear stress field with the square of the stress equilibrium condition being the error function. Noting that the element stress is not accurate at all points even if the displacement has been reasonably converged, the in-plane stress and its derivatives are only evaluated at their respective optimal stations inside the element during the least square fit. For instances, the first and second order quadrature points are known as the optimal stress points of four-node and nine-node quadrilaterals, respectively [17]. By generalizing shear correction factor in homogenous beam as a ratio of the transverse shear energies based on the strain derived directly from the first order Mindlin theory and the exact parabolic transverse shear stress distribution, the ratio of the transverse shear energies computed by the predictor transverse shear strain and the corrector transverse shear stress in each element is calculated. The corrector displacement is calculated by incorporating the shear correction factor in the element shear stiffness matrix and solving the new system equation. In testing the efficacy of the suggested procedures, standard first, second and third order Mindlin nine-node plate finite elements are considered. The accuracy of the corrector deflection and the corrector transverse shear stress are found to be satisfactory.

\section{MINDLIN PLATE FINITE ELEMENT MODELS}

Figure 1 shows the cross-section of a $N$-ply laminated plate. Without sacrificing generality, the $x-y$ plane is taken to be mid-plane. The $\mathrm{i}$-th ply counting from the bottom is bounded by $z=Z_{i}$ and $z=$ $Z_{i+1}$. In the $M$-th order Mindlin theory, the displacement field can be expressed as :

$$
\mathbf{u}=\left\{\begin{array}{c}
u \\
v \\
w
\end{array}\right\}=\left\{\begin{array}{c}
u_{0}+z u_{1}+\cdots+z^{M} u_{M} \\
v_{0}+z v_{1}+\cdots+z^{M} v_{M} \\
w_{0}
\end{array}\right\}=\left[\begin{array}{ccc:ccc:c}
1 & \cdots & z^{M} & 0 & 0 & 0 & 0 \\
0 & 0 & 0 & 1 & \cdots & z^{M} & 0 \\
0 & 0 & 0 & 0 & 0 & 0 & 1
\end{array}\right]\left\{\begin{array}{c}
u_{0} \\
\vdots \\
u_{M} \\
\hdashline v_{0} \\
\vdots \\
v_{M} \\
\hdashline w_{0}
\end{array}\right\}
$$

where the displacement parameters $u_{i}^{\prime}$ 's, $v_{i}$ 's and $w_{0}$ are functions of $x$ and $y$. The in-plane strain and 
the transverse shear strain can be derived from the displacement as :

$$
\boldsymbol{\epsilon}=\left\{\begin{array}{c}
\varepsilon_{x} \\
\varepsilon_{y} \\
2 \varepsilon_{x y}
\end{array}\right\}=\mathbf{L}_{\varepsilon} \mathbf{u}=\left[\begin{array}{ccc}
\partial / \partial x & 0 & 0 \\
0 & \partial / \partial y & 0 \\
\partial / \partial y & \partial / \partial x & 0
\end{array}\right]\left\{\begin{array}{l}
u \\
v \\
w
\end{array}\right\}, \boldsymbol{\gamma}=\left\{\begin{array}{l}
\gamma_{z x} \\
\gamma_{z y}
\end{array}\right\}=\mathbf{L}_{\gamma} \mathbf{u}=\left[\begin{array}{ccc}
\partial / \partial z & 0 & \partial / \partial x \\
0 & \partial / \partial z & \partial / \partial y
\end{array}\right]\left\{\begin{array}{l}
u \\
v \\
w
\end{array}\right\}
$$

In finite element formulation, the displacement field is obtained by nodal interpolation. For a $m$ node element, the displacement parameters are interpolated as :

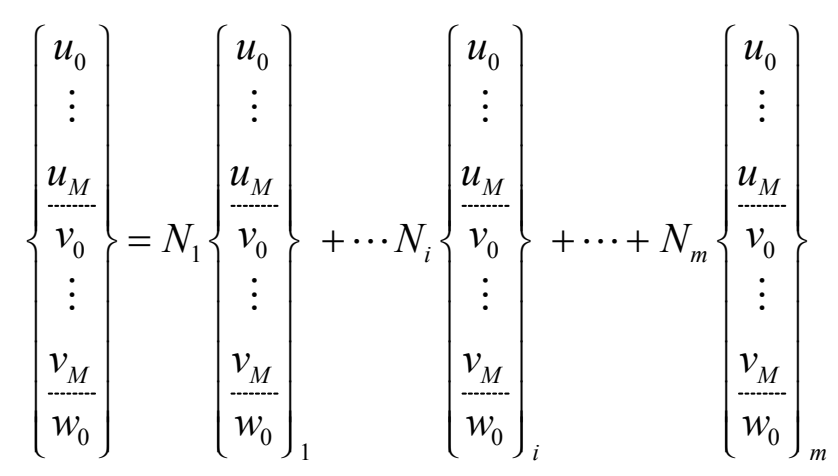

in which $N_{\mathrm{i}}$ is the interpolation function of the $i$-th node and \{\}$_{i}$ refers to the set of displacement parameters at the i-th node. By combining Eqn.(1) and Eqn.(3), the displacement field can be expressed as:

$$
\mathbf{u}=\mathbb{N} \mathbf{q}
$$

where $\mathbb{N}$ is the interpolation matrix and $\mathbf{q}$ is the vector containing all the element nodal parameters. The element strains derived from the Eqn.(4) via Eqn.(2) are:

$$
\boldsymbol{\epsilon}=\left(\mathbf{L}_{\varepsilon} \mathbb{N}\right) \mathbf{q}=\mathbb{B}_{\varepsilon} \mathbf{q} \quad \text { and } \quad \boldsymbol{\gamma}=\left(\mathbf{L}_{\gamma} \mathbb{N}\right) \mathbf{q}=\mathbb{B}_{\gamma} \mathbf{q}
$$

After incorporating the plane stress condition along the thickness direction, the constitutive relation regardless of the fibre angle can be written as:

$$
\boldsymbol{\sigma}=\left\{\begin{array}{c}
\sigma_{x} \\
\sigma_{y} \\
\sigma_{x y}
\end{array}\right\}=\mathbf{C}_{\varepsilon} \boldsymbol{\epsilon}=\mathbf{C}_{\varepsilon} \mathbb{B}_{\varepsilon} \mathbf{q} \text { and } \boldsymbol{\tau}=\left\{\begin{array}{c}
\tau_{z x} \\
\tau_{z y}
\end{array}\right\}=\mathbf{C}_{\gamma} \boldsymbol{\gamma}=\mathbf{C}_{\gamma} \mathbb{B}_{\gamma} \mathbf{q}
$$

where $\mathbf{C}_{\varepsilon}$ is the in-plane stiffness matrix and $\mathbf{C}_{\gamma}$ is the transverse shear stiffness matrix. It should be remarked that

$$
\mathbf{C}_{\varepsilon}=\mathbf{C}_{\varepsilon}^{i}, \mathbf{C}_{\gamma}=\mathbf{C}_{\gamma}^{i} \quad \text { for } z \in\left[Z_{i}, Z_{i+1}\right]
$$

in which the superscript $i$ designates the i-th ply. The strain energy stored in the element can be computed as: 


$$
U^{e}=\frac{1}{2} \mathbf{q}^{T}\left(\mathbb{K}_{\varepsilon}^{e}+\mathbb{K}_{\gamma}^{e}\right) \mathbf{q}
$$

in which

$$
\begin{aligned}
& \mathbb{K}_{\varepsilon}^{e}=\int_{A^{e}}\left(\int_{-h / 2}^{+h / 2} \mathbb{B}_{\varepsilon}^{T} \mathbf{C}_{\varepsilon} \mathbb{B}_{\varepsilon} d z\right) d A \text { is the element in-plane stiffness matrix } \\
& \mathbb{K}_{\gamma}^{e}=\int_{A^{e}}\left(\int_{-h / 2}^{+h / 2} \mathbb{B}_{\gamma}^{T} \mathbf{C}_{\gamma} \mathbb{B}_{\gamma} d z\right) d A \text { is the element shear stiffness matrix } \\
& A^{e} \text { is the planar area of the element }
\end{aligned}
$$

By assembling the element stiffness matrices and taking into account of the boundary conditions, the nodal d.o.f.s can be solved. From the latter, the predictor displacement, the predictor strain and the predictor stress can be obtained by Eqn.(4) to Eqn.(6).

\section{CORRECTOR TRANSVERSE SHEAR STRESSES}

While the classical and Mindlin plate theories can yield moderately accurate displacement, in-plane stress and strain, the transverse shear stress derived from Eqn.(6) is often unsatisfactory. A common practice for obtaining a more accurate or corrector transverse shear stress $\left(\tilde{\tau}_{z x}\right.$ and $\left.\tilde{\tau}_{z y}\right)$ is to integrate the homogeneous stress equilibrium conditions with respect to the thickness coordinate, i.e.

$$
\tilde{\tau}_{z x}=-\int_{-h / 2}^{z}\left(\frac{\partial \sigma_{x}}{\partial x}+\frac{\partial \sigma_{x y}}{\partial y}\right) d z+c_{1}(x, y) \quad \text { and } \quad \tilde{\tau}_{z y}=-\int_{-h / 2}^{z}\left(\frac{\partial \sigma_{x y}}{\partial x}+\frac{\partial \sigma_{y}}{\partial y}\right) d z+c_{2}(x, y)
$$

where c's are normally determined from the zero shear traction condition at the bottom surface of the plate (see Fig.1), i.e.

$$
\left.\tilde{\tau}_{z x}\right|_{z=-h / 2}=\left.\tilde{\tau}_{z y}\right|_{z=-h / 2}=0
$$

As the in-plane stress is only a finite element or approximate solution, it may happen that the transverse shear stress obtained from Eqn.(9) does not satisfy the zero shear traction condition at the top surface of the plate, i.e.

$$
\left.\tilde{\tau}_{z x}\right|_{z=+h / 2}=\left.\tilde{\tau}_{z y}\right|_{z=+h / 2}=0
$$

To avoid the possible inconsistency, least error fit will be employed in the present work. Along the thickness direction, each ply is divided into $n$ equal intervals, see Figure 2. Within each ply, the corrector transverse shear stresses $\tilde{\tau}_{z x}$ is obtained by using the Lagrangian interpolation functions, i.e. 


$$
\left.(\tilde{\tau})\right|_{z \in\left[Z_{1}, Z_{2}\right]}=\mathbb{M}\left\{\begin{array}{c}
\tilde{\tau}_{z x}^{1}=0 \\
\tilde{\tau}_{z x}^{1,1} \\
\vdots \\
\tilde{\tau}_{z x}^{1, n-1} \\
\tilde{\tau}_{z x}^{2}
\end{array}\right\}, \ldots,\left.(\tilde{\tau})\right|_{z \in\left[Z_{i}, Z_{i+1}\right]}=\mathbb{M}\left\{\begin{array}{c}
\tilde{\tau}_{z x}^{i} \\
\tilde{\tau}_{z x}^{i, 1} \\
\vdots \\
\tilde{\tau}_{z x}^{i, n-1} \\
\tilde{\tau}_{z x}^{i+1}
\end{array}\right\}, \ldots,\left.(\tilde{\tau})\right|_{z \in\left[Z_{N}, Z_{N+1}\right]}=\mathbb{M}\left\{\begin{array}{c}
\tilde{\tau}_{z x}^{N} \\
\tilde{\tau}_{z x}^{N, 1} \\
\vdots \\
\tilde{\tau}_{z x}^{N, n-1} \\
\tilde{\tau}_{z x}^{N+1}=0
\end{array}\right\}
$$

where

$\mathbb{M}$ is the row matrix containing the $n+1$ Lagrangian interpolation functions, $\tilde{\tau}_{z x}^{i}=\left.\tilde{\tau}_{z x}\right|_{z=Z_{i}}$ $\tilde{\tau}_{z x}^{1}$ and $\tilde{\tau}_{z x}^{N+1}$ are set to zero to satisfy the zero shear traction conditions

By collecting all the unknown $\tilde{\tau}_{z x}^{i}$ 's and $\tilde{\tau}_{z x}^{i, j}$ 's in the vector $\boldsymbol{\alpha}_{x}$, the corrector stress can be expressed symbolically as:

$$
\tilde{\tau}_{z x}=\tilde{M} \boldsymbol{\alpha}_{x} \quad \text { for } z \in\left[Z_{1}, Z_{N+1}\right]
$$

Similarly, we can set up the interpolation for $\tilde{\tau}_{z y}$ as:

$$
\tilde{\tau}_{z y}=\tilde{M} \boldsymbol{\alpha}_{y} \quad \text { for } z \in\left[Z_{1}, Z_{N+1}\right]
$$

By minimizing the following error functions with respect to $\boldsymbol{\alpha}_{x}$ and $\boldsymbol{\alpha}_{y}$ :

$$
E_{x}=\int_{-h / 2}^{+h / 2}\left(\frac{\partial \sigma_{x}}{\partial x}+\frac{\partial \sigma_{x y}}{\partial y}+\frac{\partial \tilde{\tau}_{z x}}{\partial z}\right)^{2} d z \quad, \quad E_{y}=\int_{-h / 2}^{+h / 2}\left(\frac{\partial \sigma_{x y}}{\partial x}+\frac{\partial \sigma_{y}}{\partial y}+\frac{\partial \tilde{\tau}_{z y}}{\partial z}\right)^{2} d z
$$

we get

$$
\boldsymbol{\alpha}_{x}=-\mathbb{\boxplus}^{-1}\left(\int_{-h / 2}^{+h / 2} \tilde{\mathbb{M}}_{, z}^{T}\left(\frac{\partial \sigma_{x}}{\partial x}+\frac{\partial \sigma_{x y}}{\partial y}\right) d z\right), \boldsymbol{\alpha}_{y}=-\mathbb{\boxplus}^{-1}\left(\int_{-h / 2}^{+h / 2} \tilde{\mathbb{M}}_{, z}^{T}\left(\frac{\partial \sigma_{x y}}{\partial x}+\frac{\partial \sigma_{y}}{\partial y}\right) d z\right)
$$

where

$$
\mathbb{H}=\int_{-h / 2}^{+h / 2} \tilde{\mathbb{M}},_{z}^{T} \tilde{\mathbb{M}}, z d z
$$

For the same lamination, $\mathbb{H}, \tilde{\mathbb{M}}$ and $\tilde{\mathbb{M}}, z$ are independent of the planar coordinates $(x, y)$ and applicable to all elements modelling the plate. Eqn.(1) and Eqn.(2) indicate that the in-plane stress/strain components are $M$-th polynomial of $z$ for the $M$-th order Mindlin theory. Hence, the transverse shear stress should be a (M+1)-th order polynomial of $z$ as noted in Eqn.(9). Thus, it is sufficient to keep the number of intervals $n$ in each ply (see Eqn.(12)) at $M+1$.

\section{SHEAR CORRECTION FACTOR AND CORRECTOR DISPLACEMENT}

The well-known shear correction factor for first order Mindlin theory is derived by referring to a 
section of a unit width rectangular beam made of isotropic material, see Fig.3. When the section carry a shear force $Q_{x}$, the analytical transverse shear stress and transverse shear energy are :

$$
\tilde{\tau}=\frac{3 Q_{x}}{2 h}\left(1-\frac{4 z^{2}}{h^{2}}\right) \text { and } \tilde{U}_{\text {shear }}=\frac{1}{2} \int_{-h / 2}^{+h / 2} \frac{\tilde{\tau}^{2}}{G} d z=\frac{Q_{x}^{2}}{2 G h} \frac{6}{5}
$$

where $G$ is the shear modulus. Under the first order Mindlin theory, the transverse shear strain and thus the transverse stress are constant along the thickness direction. To sustain the shear force, $\tau=Q_{x} / h$ or $\gamma=Q_{x} / G h$ and the related strain energy is :

$$
U_{\text {shear }}=\frac{1}{2} \int_{-h / 2}^{+h / 2} G \gamma^{2} d z=\frac{Q_{x}^{2}}{2 G h}
$$

To balance the two energies, a shear correction factor $k$ given as:

$$
k=U_{\text {shear }} / \tilde{U}_{\text {shear }}=5 / 6
$$

is introduced as a multiplier to $G$ such that the equality of $U_{\text {shear }}$ and $\tilde{U}_{\text {shear }}$ holds. The element stiffness matrix in Eqn.(8) which is based on strain energy calculation is modified to :

$$
\mathbb{K}^{e}=\mathbb{K}_{\varepsilon}^{e}+k \mathbb{K}_{\gamma}^{e}
$$

so as to reduce the error induced by the inconsistent stress distribution of the first order Mindlin theory.

While a priori shear correction factor for isotropic beam can be derived from the above simple argument, the latter cannot be readily generalized to laminated plates and the higher order Mindlin theory for at least two reasons. Under the higher order theory, the shear stress and strain are no more constant and their distribution in terms of the shear force is not known as a priori. Moreover, different combinations of shear force components would result in different shear correction factors.

The method proposed here for calculating shear correction factor is a posteriori and a direct generalization of Eqn.(19). It is taken to be the following ratio of the transverse shear energies computed by the predictor transverse shear strain $\boldsymbol{\gamma}$ and the corrector transverse shear stress $\tilde{\boldsymbol{\tau}}$ :

$$
k=\frac{U_{\text {shear }}}{\tilde{U}_{\text {shear }}}=\frac{\int_{-h / 2}^{+h / 2}\left(\boldsymbol{\gamma}^{T} \mathbf{C}_{\gamma} \boldsymbol{\gamma}\right) d z}{\int_{-h / 2}^{+h / 2}\left(\tilde{\boldsymbol{\tau}}^{T} \mathbf{C}_{\gamma}^{-1} \tilde{\boldsymbol{\tau}}\right) d z}
$$

With the above $k$ substituted into Eqn.(20), a new system matrix is formed and used to compute the corrector displacement. 


\section{IMPLEMENTATION IN NINE-NODE MINDLIN PLATE ELEMENTS}

Restricting ourselves to rectangular elements with its parametric $\xi$-and $\eta$-axes parallel to the $x$ - and $y$-axes in this paragraph (see Fig.4), the interpolated displacement variables are biquadratic in $x$ and $y$. Thus, the in-plane stress/strain are bilinear in $x$ and $y$ whereas the $x$ - and $y$-derivatives of the inplane stress is linear in $y$ and $x$, respectively. For consistency, the corrector transverse shear stress should be taken to be independent of $x$ and $y$ inside the element (see Eqn.(9)).

From the above argument, it would be sensible to compute the corrector transverse shear stress only at the element origin. To avoid the complications of computing the second derivatives of the displacement inside a general quadrilateral [10], the predictor in-plane stress is first computed at the four second order Gaussian stations which are well-known as the optimal stress points of the ninenode quadrilateral elements [17]. The four stresses are then interpolated as :

$$
\boldsymbol{\sigma}=\frac{(1-\tilde{\xi})(1-\tilde{\eta})}{4} \boldsymbol{\sigma}_{I}(z)+\frac{(1+\tilde{\xi})(1-\tilde{\eta})}{4} \boldsymbol{\sigma}_{I I}(z)+\frac{(1+\tilde{\xi})(1+\tilde{\eta})}{4} \boldsymbol{\sigma}_{I I I}(z)+\frac{(1-\tilde{\xi})(1+\tilde{\eta})}{4} \boldsymbol{\sigma}_{I V}(z)
$$

where $\boldsymbol{\sigma}_{i}$ is the stress at the $i$-th integration station, see Fig.4, $\tilde{\xi}=\xi \sqrt{3}$ and $\tilde{\eta}=\eta \sqrt{3}$. The derivatives of the stress at the element origin are obtained from the above interpolation as :

$$
\begin{aligned}
& \frac{\partial \boldsymbol{\sigma}}{\partial x}=\left.\frac{\partial \xi}{\partial x}\right|_{\xi=\eta=0} \frac{\sqrt{3}}{4}\left(-\boldsymbol{\sigma}_{I}+\boldsymbol{\sigma}_{I I}+\boldsymbol{\sigma}_{I I I}-\boldsymbol{\sigma}_{I V}\right)+\left.\frac{\partial \eta}{\partial x}\right|_{\xi=\eta=0} \frac{\sqrt{3}}{4}\left(-\boldsymbol{\sigma}_{I}-\boldsymbol{\sigma}_{I I}+\boldsymbol{\sigma}_{I I I}+\boldsymbol{\sigma}_{I V}\right) \\
& \frac{\partial \boldsymbol{\sigma}}{\partial y}=\left.\frac{\partial \xi}{\partial y}\right|_{\xi=\eta=0} \frac{\sqrt{3}}{4}\left(-\boldsymbol{\sigma}_{I}+\boldsymbol{\sigma}_{I I}+\boldsymbol{\sigma}_{I I I}-\boldsymbol{\sigma}_{I V}\right)+\left.\frac{\partial \eta}{\partial y}\right|_{\xi=\eta=0} \frac{\sqrt{3}}{4}\left(-\boldsymbol{\sigma}_{I}-\boldsymbol{\sigma}_{I I}+\boldsymbol{\sigma}_{I I I}+\boldsymbol{\sigma}_{I V}\right)
\end{aligned}
$$

where

$$
\left[\begin{array}{ll}
\left.(\partial x / \partial \xi)\right|_{\xi=\eta=0} & \left.(\partial x / \partial \eta)\right|_{\xi=\eta=0} \\
\left.(\partial y / \partial \xi)\right|_{\xi=\eta=0} & \left.(\partial y / \partial \eta)\right|_{\xi=\eta=0}
\end{array}\right]=\frac{1}{4}\left[\begin{array}{ll}
x_{6}-x_{8} & x_{7}-x_{5} \\
y_{6}-y_{8} & y_{7}-y_{5}
\end{array}\right]^{-1}
$$

$x_{i}$ and $y_{i}$ are nodal coordinates (see Fig.4)

The corrector transverse shear stress can then be calculated according to Eqn.(13), Eqn.(14) and Eqn.(16). 


\section{NUMERICAL EXAMPLES}

In this section, simply-supported laminated plates loaded by sinusoidal distributed forces will be considered. To assess the accuracy of the corrector deflection and the corrector transverse shear stress, they are compared with the three-dimensional elasticity solutions obtained by Pagano [1819]. Following Pagano's choice of material constants, we have

$$
E_{L} / E_{T}=25, G_{L T} / E_{T}=0.5, G_{T T} / E_{T}=0.2, v_{T T}=v_{L T}=0.25
$$

in which $L$ and $T$ stand for the longitudinal (fibre) and transverse directions, respectively. The first, second and third order Mindlin nine-node plate elements are employed. All these elements are evaluated by the second order quadrature along the in-plane directions.

\subsection{Cylindrical Bending Problems}

In this series of problems, the dimensions of the plates along the $x$-, $y$-, and $z$-directions are respectively $l, \infty$ and $h$, see Fig.5. The plane strain condition is assumed along the $y$-direction. The plates are simply supported along $x=0$ and $x=l$. The downward sinusoidal transverse distributed force :

$$
q=q_{0} \sin (\pi x / l)
$$

is prescribed. Two-ply $\left(0^{0} / 90^{0}\right)$, three-ply $\left(0^{0} / 90^{0} / 0^{0}\right)$, six-ply $\left(0^{0} / 90^{0} / 0^{0} / 90^{0} / 0^{0} / 90^{0}\right)$ and seven-ply $\left(0^{0} / 90^{0} / 0^{0} / 90^{\circ} / 0^{0} / 90^{\circ} / 0^{0}\right)$ cross-laminated plates are considered. For symmetric laminations (odd number of plies) with the number of plies larger than 3 , the total thickness of the $0^{0}$ and $90^{0}$ plies are the same whereas layers with the same fibre angles are of equal thickness. For non-symmetric laminations (even number of plies) and 3-ply lamination, all plies are of equal thickness. These thickness distributions also apply to the problems in Section 6.2. The plates are modelled by eight elements along the x-direction and their aspect ratios $S=l / h$ are varied from 4 to 100 . Accuracy of the computed results remain practically constant even if more elements are employed. The predictor and corrector midspan deflections are listed in Table 1. For symmetric plates, the first and second order Mindlin models always yield identical results. The improvement of the corrector deflections over the predictor deflections is most obvious for $S=4,10$ and 20. Fig.6 to Fig.13 depict the thickness variation of the corrector transverse shear stress for $S=4$ and 10 at $\mathrm{x}=l / 16$ which is the center of the element at the left hand support. Satisfactory stresses are obtained for $S=10$. The accuracy increases with $S$ and becomes highly accurate for $S \geq 20$ whose results are not shown due to the limitation of space. Moreover, the improvement of the higher order Mindlin theories over the first order one is apparent only for $S=4$. 
Table1. Percentage errors of the predictor and corrector midspan deflections for cross-laminated plates under cylindrical bending.

\begin{tabular}{|c|c|c|c|c|c|c|c|c|c|}
\hline & \multicolumn{2}{|c|}{ 2-ply } & \multicolumn{2}{|c|}{ 3-ply } & \multicolumn{2}{|c|}{ 6-ply } & \multicolumn{2}{|c|}{ 7-ply } \\
\hline & & predictor & orrector & predictor & orrector & predictor & corrector & predictor & corrector \\
\hline \multirow{4}{*}{$\begin{array}{c}S= \\
4\end{array}$} & $1^{\text {st }}$ order & $-5.69 \%$ & $1.45 \%$ & $-27.30 \%$ & $9.52 \%$ & $-26.99 \%$ & $1.42 \%$ & $-23.14 \%$ & $0.42 \%$ \\
\hline & $2^{\text {nd }}$ order & $-4.45 \%$ & $1.04 \%$ & $-27.30 \%$ & $9.52 \%$ & $-26.27 \%$ & $1.50 \%$ & $-23.14 \%$ & $0.42 \%$ \\
\hline & $3^{\text {rd }}$ order & $-1.02 \%$ & $0.87 \%$ & $-6.01 \%$ & $0.14 \%$ & $-13.75 \%$ & $-0.10 \%$ & $-14.41 \%$ & $-0.67 \%$ \\
\hline & exact & 0.0469 & ${ }_{0} l^{4} / E_{\mathrm{T}} h^{3}$ & $0.02897 q$ & $l^{4} / E_{\mathrm{T}} h^{3}$ & $0.03872 q$ & ${ }_{0} l^{4} / E_{\mathrm{T}} h^{3}$ & 0.03310 & ${ }_{0} l^{4} / E_{\mathrm{T}} h^{3}$ \\
\hline \multirow{4}{*}{$\begin{array}{l}S= \\
10\end{array}$} & $1^{\text {st }}$ order & $-1.56 \%$ & $0.27 \%$ & $-18.05 \%$ & $0.22 \%$ & $-12.22 \%$ & $-0.40 \%$ & $-11.24 \%$ & $-0.69 \%$ \\
\hline & $2^{\text {nd }}$ order & $-1.19 \%$ & $0.24 \%$ & $-18.05 \%$ & $0.22 \%$ & $-11.89 \%$ & $-0.40 \%$ & $-11.24 \%$ & $-0.69 \%$ \\
\hline & $3^{\text {rd }}$ order & $-0.31 \%$ & $0.24 \%$ & $-5.37 \%$ & $-0.75 \%$ & $-6.45 \%$ & $-0.54 \%$ & $-6.95 \%$ & $-0.77 \%$ \\
\hline & exact & 0.0295 & ${ }_{0} l^{4} / E_{\mathrm{T}} h^{3}$ & $0.00931 q$ & $l^{4} / E_{\mathrm{T}} h^{3}$ & $0.01489 q$ & ${ }_{0} l^{4} / E_{\mathrm{T}} h^{3}$ & 0.01165 & ${ }_{0} l^{4} / E_{\mathrm{T}} h^{3}$ \\
\hline \multirow{4}{*}{$\begin{array}{c}S= \\
15\end{array}$} & $1^{\text {st }}$ order & $-0.74 \%$ & $0.12 \%$ & $-11.10 \%$ & $-0.33 \%$ & $-6.66 \%$ & $-0.29 \%$ & $-6.30 \%$ & $-0.43 \%$ \\
\hline & $2^{\text {nd }}$ order & $-0.56 \%$ & $0.12 \%$ & $-11.10 \%$ & $-0.33 \%$ & $-6.46 \%$ & $-0.29 \%$ & $-6.30 \%$ & $-0.43 \%$ \\
\hline & $3^{\text {rd }}$ order & $-0.14 \%$ & $0.11 \%$ & $-3.46 \%$ & $-0.58 \%$ & $-3.53 \%$ & $-0.32 \%$ & $-3.88 \%$ & $-0.46 \%$ \\
\hline & exact & 0.0276 & ${ }_{0} l^{4} / E_{\mathrm{T}} h^{3}$ & $0.00700 q$ & $l^{4} / E_{\mathrm{T}} h^{3}$ & $0.01228 q$ & ${ }_{0} l^{4} / E_{\mathrm{T}} h^{3}$ & 0.00932 & ${ }_{0} l^{4} / E_{\mathrm{T}} h^{3}$ \\
\hline \multirow{4}{*}{$\begin{array}{l}S= \\
20\end{array}$} & $1^{\text {st }}$ order & $-0.44 \%$ & $0.07 \%$ & $-7.13 \%$ & $-0.32 \%$ & $-4.05 \%$ & $-0.18 \%$ & $-3.88 \%$ & $-0.24 \%$ \\
\hline & $2^{\text {nd }}$ order & $-0.33 \%$ & $0.04 \%$ & $-7.13 \%$ & $-0.32 \%$ & $-3.96 \%$ & $-0.18 \%$ & $-3.88 \%$ & $-0.24 \%$ \\
\hline & $3^{\text {rd }}$ order & $-0.11 \%$ & $0.04 \%$ & $-2.27 \%$ & $-0.32 \%$ & $-2.11 \%$ & $-0.18 \%$ & $-2.35 \%$ & $-0.24 \%$ \\
\hline & exact & 0.0270 & $l^{4} / E_{\mathrm{T}} h^{3}$ & $0.00617 q$ & $l^{4} / E_{\mathrm{T}} h^{3}$ & $0.01136 q$ & ${ }_{0} l^{4} / E_{\mathrm{T}} h^{3}$ & 0.00850 & ${ }_{0} l^{4} / E_{\mathrm{T}} h^{3}$ \\
\hline \multirow{4}{*}{$\begin{array}{c}S= \\
50\end{array}$} & $1^{\text {st }}$ order & $-0.08 \%$ & $0.00 \%$ & $-1.33 \%$ & $0.00 \%$ & $-0.68 \%$ & $0.00 \%$ & $-0.79 \%$ & $-0.13 \%$ \\
\hline & $2^{\text {nd }}$ order & $-0.04 \%$ & $0.00 \%$ & $-1.33 \%$ & $0.00 \%$ & $-0.68 \%$ & $0.00 \%$ & $-0.79 \%$ & $-0.13 \%$ \\
\hline & $3^{\text {rd }}$ order & $0.00 \%$ & $0.00 \%$ & $-0.38 \%$ & $0.00 \%$ & $-0.29 \%$ & $0.00 \%$ & $-0.53 \%$ & $-0.13 \%$ \\
\hline & exact & 0.0263 & $l^{4} / E_{\mathrm{T}} h^{3}$ & $0.00527 q$ & $l^{4} / E_{\mathrm{T}} h^{3}$ & $0.01036 q$ & ${ }_{0} l^{4} / E_{\mathrm{T}} h^{3}$ & 0.00762 & ${ }_{0} l^{4} / E_{\mathrm{T}} h^{3}$ \\
\hline \multirow{4}{*}{$\begin{array}{l}S= \\
100\end{array}$} & $1^{\text {st }}$ order & $0.00 \%$ & $0.00 \%$ & $-0.39 \%$ & $0.00 \%$ & $-0.20 \%$ & $0.00 \%$ & $-0.13 \%$ & $0.00 \%$ \\
\hline & $2^{\text {nd }}$ order & $0.00 \%$ & $0.00 \%$ & $-0.39 \%$ & $0.00 \%$ & $-0.20 \%$ & $0.00 \%$ & $-0.13 \%$ & $0.00 \%$ \\
\hline & $3^{\text {rd }}$ order & $0.00 \%$ & $0.00 \%$ & $-0.20 \%$ & $0.00 \%$ & $-0.10 \%$ & $0.00 \%$ & $-0.13 \%$ & $0.00 \%$ \\
\hline & & 0.0262 & ${ }^{4} / E_{\mathrm{T}} h^{3}$ & $0.00514 q$ & $l^{4} / E_{\mathrm{T}} h^{3}$ & $0.01022 q$ & ${ }_{0} l^{4} / E_{\mathrm{T}} h^{3}$ & 0.00749 & ${ }_{0} l^{4} / E_{\mathrm{T}} h^{3}$ \\
\hline
\end{tabular}

\subsection{Square Plate Problems}

In this series of problems, the dimensions of the plate along the $x$-, $y$-, and $z$-directions are $l, l$ and $h$, respectively. The plate is simply supported along its four edges. The downward double sinusoidal transverse distributed force :

$$
q=q_{0} \sin (\pi x / l) \sin (\pi y / l)
$$

is prescribed. Two-ply $\left(0^{0} / 90^{0}\right)$, three-ply $\left(0^{0} / 90^{0} / 0^{0}\right)$, four-ply $\left(0^{0} / 90^{\circ} / 0^{0} / 90^{0}\right)$, five-ply $\left(0^{0} / 90^{0} /\right.$ $\left.0^{0} / 90^{0} / 0^{0}\right)$, six-ply $\left(0^{0} / 90^{\circ} / 0^{0} / 90^{\circ} / 0^{0} / 90^{0}\right)$, seven-ply $\left(0^{0} / 90^{\circ} / 0^{0} / 90^{0} / 0^{\circ} / 90^{0} / 0^{0}\right)$, eight-ply $\left(0^{0} / 90^{0} / 0^{0} /\right.$ $\left.90^{0} / 0^{0} / 90^{0} / 0^{0} / 90^{0}\right)$ and nine-ply $\left(0^{0} / 90^{0} / 0^{0} / 90^{0} / 0^{0} / 90^{0} / 0^{0} / 90^{0} / 0^{0}\right)$ cross-laminated plates are considered. The plates are modelled by $8 \times 8$ elements and their aspect ratios $S=l / h$ are varied from 
4 to 100 . Accuracy of the computed results remain practically constant even if more elements are employed. The predictor and corrector midspan deflections are listed in Tables 2 and 3. The improvement of the corrector deflections over the predictor deflections is obvious for $S \leq 50$. Fig.14 to Fig.25 depict the thickness variation of the corrector $\tau_{z x}$ at $(x=l / 32, y=15 l / 32)$ and the corrector $\tau_{z y}$ at $(x=15 l / 32, y=l / 32)$ for $S=10$. The two points are the element centroids closest to mid-edge points of the plate. The accuracy of the corrector transverse shear stress appears to be acceptable and the difference between different Mindlin theories is insignificant. Same as the problems in the last subsection, the accuracy increases and the difference reduces as $S$ goes up. To illustrate this point, we first note that the stress predictions for the 3-ply plates shown in Fig.15 and Fig16 are least satisfactory among all. Apparent improvement in accuracy can be observed when $S$ is increased to 15 as depicted in Fig.26 and Fig.27. For non-symmetric plates, the $\tau_{z x}$-variations from $z=-h / 2$ to $z=+h / 2$ at $(x=l / 32, y=15 l / 32)$ is identical to $\tau_{z y}$-variations from $z=+h / 2$ to $z=-h / 2$ at $(x=15 l / 32, y=l / 32)$. Hence, the latter variations are skipped.

Table 2. Percentage errors of the predictor and corrector midspan deflections for cross-ply symmetric laminated square plates under double sinusoidal loading.

\begin{tabular}{|c|c|c|c|c|c|}
\hline & \multirow{2}{*}{$\begin{array}{l}\text { 3-ply } \\
\text { predictor corrector }\end{array}$} & 5-ply & 7-ply & 9-ply \\
\hline & & & predictor corrector & predictor corrector & predictor corrector \\
\hline & $1^{\text {st }} / 2^{\text {nd }}$ order & $-21.83 \% \quad 7.93 \%$ & $-25.52 \% \quad 4.03 \%$ & $-24.30 \% \quad 2.43 \%$ & $-23.39 \%$ \\
\hline$S=$ & $3^{\text {rd }}$ order & $-3.99 \% \quad 0.05 \%$ & $-12.44 \%$ & $-13.10 \%$ & $-13.04 \%$ \\
\hline 4 & exact & $0.02006 q_{0} a^{4} / E_{\mathrm{T}} h^{3}$ & $0.01850 q_{0} a^{4} / E_{\mathrm{T}} h^{3}$ & $0.01791 q_{0} a^{4} / E_{\mathrm{T}} h^{3}$ & $0.01759 q_{0} a^{4} / E_{\mathrm{T}} h^{3}$ \\
\hline & $1^{\text {st }} / 2^{\text {nd }}$ order & $-16.20 \%-0.13 \%$ & $-12.87 \% \quad-0.57 \%$ & $-11.64 \% \quad-0.39 \%$ & $-11.04 \% \quad-0.33 \%$ \\
\hline$S=$ & $3^{\text {rd }}$ order & $-4.65 \% \quad-1.06 \%$ & $-6.88 \quad \% \quad-0.57 \%$ & $-6.48 \quad \% \quad-0.46 \%$ & $-6.22 \% \quad-0.40 \%$ \\
\hline 10 & exact & $0.00753 q_{0} a^{4} / E_{\mathrm{T}} h^{3}$ & $0.00677 q_{0} a^{4} / E_{\mathrm{T}} h^{3}$ & $0.00659 q_{0} a^{4} / E_{\mathrm{T}} h^{3}$ & $0.00652 q_{0} a^{4} / E_{\mathrm{T}} h^{3}$ \\
\hline & $1^{\text {st }} / 2^{\text {nd }}$ order & $-10.19 \% \quad 0.72 \%$ & $-7.28 \%$ & $-6.47 \%$ & $-6.11 \%$ \\
\hline$S=$ & $3^{\text {rd }}$ order & $-3.10 \quad \% \quad-0.78 \%$ & $-3.98 \% \quad-0.47 \%$ & $-3.63 \%$ & $-0.29 \%$ \\
\hline 15 & exact & $0.00580 q_{0} a^{4} / E_{\mathrm{T}} h^{3}$ & $0.00542 q_{0} a^{4} / E_{\mathrm{T}} h^{3}$ & $0.00534 q_{0} a^{4} / E_{\mathrm{T}} h^{3}$ & $0.00530 q_{0} a^{4} / E_{\mathrm{T}} h^{3}$ \\
\hline & $1^{\text {st }} / 2^{\text {nd }}$ order & $-6.59 \% \quad-0.39 \%$ & $-4.45 \%$ & $-3.97 \%$ & $-0.18 \%$ \\
\hline$S=$ & $3^{\text {rd }}$ order & $-1.94 \%$ & $-2.45 \%$ & $-2.29 \%$ & $-0.18 \%$ \\
\hline 20 & exact & $0.00516 q_{0} a^{4} / E_{\mathrm{T}} h^{3}$ & $0.00494 q_{0} a^{4} / E_{\mathrm{T}} h^{3}$ & $0.00489 q_{0} a^{4} / E_{\mathrm{T}} h^{3}$ & $0.00487 q_{0} a^{4} / E_{\mathrm{T}} h^{3}$ \\
\hline & $1^{\text {st }} / 2^{\text {nd }}$ order & $-1.35 \% \quad 0.00 \%$ & $-0.78 \%$ & $-0.69 \%$ & $-0.10 \%$ \\
\hline$S=$ & $3^{\text {rd }}$ order & $-0.45 \% \quad 0.00 \%$ & $-0.39 \%$ & $-0.39 \%$ & $-0.39 \%$ \\
\hline 50 & exact & $0.00445 q_{0} a^{4} / E_{\mathrm{T}} h^{3}$ & $0.0441 q_{0} a^{4} / E_{\mathrm{T}} h^{3}$ & $0.00441 q_{0} a^{4} / E_{\mathrm{T}} h^{3}$ & $0.00440 q_{0} a^{4} / E_{\mathrm{T}} h^{3}$ \\
\hline & $1^{\text {st }} / 2^{\text {nd }}$ order & $-0.46 \% \quad 0.00 \%$ & $-0.20 \%$ & $-0.10 \%$ & $-0.20 \%$ \\
\hline$S=$ & $3^{\text {rd }}$ order & $-0.23 \% \quad 0.00 \%$ & $-0.10 \%$ & $-0.10 \%$ & $-0.10 \%$ \\
\hline 100 & exact & $0.00435 q_{0} a^{4} / E_{\mathrm{T}} h^{3}$ & $0.00434 q_{0} a^{4} / E_{\mathrm{T}} h^{3}$ & $0.00433 q_{0} a^{4} / E_{\mathrm{T}} h^{3}$ & $0.00433 q_{0} a^{4} / E_{\mathrm{T}} h^{3}$ \\
\hline
\end{tabular}


Table 3. Percentage errors of the predictor and corrector midspan deflections for cross-ply nonsymmetric laminated square plates under double sinusoidal loading.

\begin{tabular}{|c|c|c|c|c|c|c|c|c|c|}
\hline & \multicolumn{2}{|c|}{ 2-ply } & \multicolumn{2}{|c|}{ 4-ply } & \multicolumn{2}{|c|}{ 6-ply } & \multicolumn{2}{|c|}{ 8-ply } \\
\hline & & predicto & corrector & predictor & corrector & predictor & corrector & predictor & corrector \\
\hline \multirow{4}{*}{$\begin{array}{c}S= \\
4\end{array}$} & $1^{\text {st }}$ order & $-4.84 \%$ & $1.50 \%$ & $-27.95 \%$ & $8.31 \%$ & $-26.02 \%$ & $3.48 \%$ & $-24.45 \%$ & $2.55 \%$ \\
\hline & $2^{\text {nd }}$ order & $-4.59 \%$ & $1.45 \%$ & $-26.84 \%$ & $8.17 \%$ & $-25.48 \%$ & $3.55 \%$ & $-24.14 \%$ & $2.65 \%$ \\
\hline & $3^{\text {rd }}$ order & $-0.63 \%$ & $1.26 \%$ & $-12.33 \%$ & $3.33 \%$ & $-13.15 \%$ & $1.61 \%$ & $-13.15 \%$ & $1.08 \%$ \\
\hline & exact & 0.02068 & $a^{4} / E_{\mathrm{T}} h^{3}$ & $0.01958 q$ & $a^{4} / E_{\mathrm{T}} h^{3}$ & $0.01847 q$ & $a^{4} / E_{\mathrm{T}} h^{3}$ & $0.01790 q$ & $a^{4} / E_{\mathrm{T}} h^{3}$ \\
\hline \multirow{4}{*}{$\begin{array}{c}S= \\
10\end{array}$} & $1^{\text {st }}$ order & $-1.55 \%$ & $0.16 \%$ & $-14.59 \%$ & $0.51 \%$ & $-12.51 \%$ & $0.06 \%$ & $-11.53 \%$ & $0.00 \%$ \\
\hline & $2^{\text {nd }}$ order & $-1.47 \%$ & $0.16 \%$ & $-14.08 \%$ & $0.51 \%$ & $-12.20 \%$ & $0.06 \%$ & $-11.33 \%$ & $0.06 \%$ \\
\hline & $3^{\text {rd }}$ order & $-0.33 \%$ & $0.16 \%$ & $-7.52 \%$ & $0.17 \%$ & $-6.60 \%$ & $-0.06 \%$ & $-6.25 \%$ & $-0.06 \%$ \\
\hline & exact & 0.01227 & $a^{4} / E_{\mathrm{T}} h^{3}$ & $0.00762 q$ & $a^{4} / E_{\mathrm{T}} h^{3}$ & $0.00693 q$ & $a^{4} / E_{\mathrm{T}} h^{3}$ & $0.00670 q$ & $a^{4} / E_{\mathrm{T}} h^{3}$ \\
\hline \multirow{4}{*}{$\begin{array}{c}S= \\
15\end{array}$} & $1^{\text {st }}$ order & $-0.76 \%$ & $0.06 \%$ & $-8.17 \%$ & $0.07 \%$ & $-6.89 \%$ & $-0.04 \%$ & $-6.34 \%$ & $-0.06 \%$ \\
\hline & $2^{\text {nd }}$ order & $-0.72 \%$ & $0.06 \%$ & $-7.89 \%$ & $0.05 \%$ & $-6.75 \%$ & $-0.04 \%$ & $-6.25 \%$ & $-0.06 \%$ \\
\hline & $3^{\text {rd }}$ order & $-0.18 \%$ & $0.06 \%$ & $-4.28 \%$ & $-0.03 \%$ & $-3.67 \%$ & $-0.07 \%$ & $-3.45 \%$ & $-0.09 \%$ \\
\hline & exact & 0.01137 & ${ }^{4} / E_{\mathrm{T}} h^{3}$ & $0.00622 q$ & $a^{4} / E_{\mathrm{T}} h^{3}$ & $0.00565 q$ & $a^{4} / E_{\mathrm{T}} h^{3}$ & $0.00547 q$ & $a^{4} / E_{\mathrm{T}} h^{3}$ \\
\hline \multirow{4}{*}{$\begin{array}{l}S= \\
20\end{array}$} & $1^{\text {st }}$ order & $-0.45 \%$ & $0.00 \%$ & $-5.06 \%$ & $0.00 \%$ & $-4.23 \%$ & $-0.08 \%$ & $-3.85 \%$ & $-0.09 \%$ \\
\hline & $2^{\text {nd }}$ order & $-0.45 \%$ & $0.00 \%$ & $-4.83 \%$ & $0.00 \%$ & $-4.15 \%$ & $-0.08 \%$ & $-3.85 \%$ & $-0.09 \%$ \\
\hline & $3^{\text {rd }}$ order & $-0.27 \%$ & $0.00 \%$ & $-2.64 \%$ & $0.00 \%$ & $-2.32 \%$ & $-0.08 \%$ & $-2.14 \%$ & $-0.09 \%$ \\
\hline & exact & 0.01105 & $a^{4} / E_{\mathrm{T}} h^{3}$ & $0.00571 q$ & $a^{4} / E_{\mathrm{T}} h^{3}$ & $0.00520 q$ & $a^{4} / E_{\mathrm{T}} h^{3}$ & $0.00504 q$ & $a^{4} / E_{\mathrm{T}} h^{3}$ \\
\hline \multirow{4}{*}{$\begin{array}{c}S= \\
50\end{array}$} & $1^{\text {st }}$ order & $-0.09 \%$ & $0.00 \%$ & $-0.92 \%$ & $0.00 \%$ & $-0.73 \%$ & $0.00 \%$ & $-0.66 \%$ & $0.00 \%$ \\
\hline & $2^{\text {nd }}$ order & $-0.09 \%$ & $0.00 \%$ & $-0.92 \%$ & $0.00 \%$ & $-0.73 \%$ & $0.00 \%$ & $-0.66 \%$ & $0.00 \%$ \\
\hline & $3^{\text {rd }}$ order & $0.00 \%$ & $0.00 \%$ & $-0.50 \%$ & $0.00 \%$ & $-0.37 \%$ & $0.00 \%$ & $-0.38 \%$ & $0.00 \%$ \\
\hline & exact & $0.0170 c$ & $4^{4} / E_{\mathrm{T}} h^{3}$ & $0.00517 q$ & $a^{4} / E_{\mathrm{T}} h^{3}$ & $0.00471 q$ & $a^{4} / E_{\mathrm{T}} h^{3}$ & $0.00457 q$ & $a^{4} / E_{\mathrm{T}} h^{3}$ \\
\hline \multirow{4}{*}{$\begin{array}{l}S= \\
100\end{array}$} & $1^{\text {st }}$ order & $0.00 \%$ & $0.00 \%$ & $-0.25 \%$ & $0.00 \%$ & $-0.19 \%$ & $0.00 \%$ & $-0.19 \%$ & $0.00 \%$ \\
\hline & $2^{\text {nd }}$ order & $0.00 \%$ & $0.00 \%$ & $-0.25 \%$ & $0.00 \%$ & $-0.19 \%$ & $0.00 \%$ & $-0.19 \%$ & $0.00 \%$ \\
\hline & $3^{\text {rd }}$ order & $0.00 \%$ & $0.00 \%$ & $-0.17 \%$ & $0.00 \%$ & $-0.09 \%$ & $0.00 \%$ & $-0.10 \%$ & $0.00 \%$ \\
\hline & & 0.01065 & ${ }^{4} / E_{\mathrm{T}} h^{3}$ & $0.00509 q$ & $4^{4} / E_{\mathrm{T}} h^{3}$ & $0.00464 q$ & $a^{4} / E_{\mathrm{T}} h^{3}$ & $0.00450 q$ & $a^{4} / E_{\mathrm{T}} h^{3}$ \\
\hline
\end{tabular}

\section{CLOSURE}

The method of integrating the equilibrium condition to obtain the transverse shear stress from the in-plane stress had been commonly adopted and also applied to many of the ad hoc finite element models for laminate analysis [4-6,9,10]. However, most of these models suffer from an increasing number of nodal parameters with the number of plies and/or non-conventional nodal kinetic d.o.f.s $[5,7,9-11,14,16]$. On the other hand, there are seldom any works that apply and detail how to apply the method to conventional finite element models such as the nine-node first order Mindlin (also known as $\mathrm{C}^{0}$ ) plate element which are available in most commercial codes. By considering the order of derivative in the strain-displacement relation and the equilibrium condition, the method can 
only be applied consistently to element models which are at least quadratic in order. Moreover, the element stress is not accurate at every point and its derivatives are even less accurate. Using the equilibrium condition again, this paper adopted a least square fit procedure to determine the corrector transverse shear stress that strictly fulfills the zero shear traction condition. To enhance the accuracy, the in-plane stress and its derivatives are only evaluated at their respective optimal stations inside the element. By generalizing shear correction factor in homogenous beams as a ratio of the transverse shear energies based on the strain derived directly from the first order Mindlin theory and the exact parabolic shear stress distribution, shear correction factor for each element are estimated and used to scale the element shear stiffness matrix.

To assess the accuracy of the proposed procedure, a large number of cross-ply laminated plate problems are examined. These problems are selected due to the availability of their threedimensional elasticity solutions. The predictor deflections, corrector deflections and corrector transverse shear stresses are reported. Based on the results, the accuracy of the proposed predictorcorrector procedures for the deflection and the transverse shear stress are found to be excellent for all aspect ratios and satisfactory for aspect ratios larger than or equal to $10 \sim 15$, respectively. An interesting observation is that for the number of laminations larger than 3 and the aspect ratio larger than or equal $10 \sim 15$, the differences between the corrector deflections and corrector transverse shear stresses computed by the various Mindlin finite element models are practically identical. These suggest that satisfactory laminate analysis may be accomplished with the first order ninenode Mindlin plate model which is available in many commercial codes and the most economic model among the three considered. Further verifications using angle-ply laminates will be necessary. The procedures devised here can readily be extended to other quadratic and higher order elements such as the six-node triangle and the sixteen-node quadrilateral.

Acknowledgment - The financial support of the University of Hong Kong in the form of a CRGC grant is gratefully acknowledged. 


\section{References}

1. E.Reissner, "The effect of transverse shear deformation on the bending of elastic plates", ASME-J.Applied Mech., 12, 69-77 (1945)

2. R.D.Mindlin, "Influence of rotary inertia and shear on flexural motions of isotropic, elastic plates", ASME-J.Applied Mech., 18, 31-38 (1951)

3. K.H.Lo, R.M.Christensen, E.M.Wu, "A high-order theory of plate deformation - Part 1 : homogeneous plates and Part 2 : laminated plates", J.Appl.Mech.-Trans.ASME, 44, 663-676 (1977)

4. J.N.Reddy, "A simple higher-order theory for laminated composite plates", J.Appl.Mech. Trans.ASME, 51, 745-752 (1984)

5. J.J.Engblom, O.O.Ochoa, "Finite element formulation including interlaminar stress calculations", Computers \& Structures, 23, 241-249 (1986)

6. A.K.Noor, W.S.Burton, "Assessment of shear deformation theories for multilayered composite plates", Applied Mechanics Review., 42, 1-12 (1989)

7. S.T.Mau, P.Tong and T.H.H.Pian, "Finite element solution for laminated thick plates", J.Composite Materials, 6, 304-311 (1972)

8. S.Srinivas, "A refined analysis of composite laminates", J.Sound \& Vibration, 30, 495-507 (1973)

9. R.A.Chaudhri, "An equilibrium method for prediction of transverse shear stresses in an thick laminated plate", Computers \& Structures, 23, 139-146 (1986)

10. J.N.Reddy, E.J.Barbero, J.L.Teply, "A plate bending element based on a generalized laminate plate theory", Int.J.Numer.Methods Engrg., 28, 2275-2292 (1989)

11. J.Han, S.V.Hoa, "A three-dimensional multilayer composite finite element for stress analysis of composite laminates”, Inter.J.Numer.Methods Eng., 36, 3903-3914 (1993)

12. M.D.Sciuva, "An improved shear-deformation theory for moderately thick multilayered anisotropic shells and plates", ASME-J. Applied Mech., 54, 589-596 (1987)

13. K.H.Lee, N.R.Senthilnathan, P.S.Lim, S.T.Chow, "An improved zig-zag model for the bending of laminated composite plates”, Composite Structures, 15, 137-148 (1990)

14. M.D.Sciuva, "A third-order triangular multilayered plate finite element with continuous interlaminar stresses", Inter.J.Numer.Methods Eng., 38, 1-26 (1995)

15. X.Li, D.Liu, "Generalized laminate theories based on double suerpothesis", Inter.J.Numer. Methods Eng., 40, 1197-1212 (1997)

16. K.Y.Sze, R.-G.Chen, Y.K.Cheung, "Finite element model with continuous transverse shear stress for composite laminates in cylindrical bending", Finite Elements in Analysis \& Design, 31, 153-164 (December 1998)

17. J.Barlow, "More on optimal stress points - reduced integration, element distortions and error estimation", Inter.J.Numer.Methods Engrg., 28, 1487-1504 (1989)

18. N.J.Pagano, "Exact solutions for composite laminates in cylindrical bending", J. Composite Material, 3, 398-411 (1969)

19. N.J.Pagano, "Exact solutions for rectangular bidirectional composite and sandwich plates", $J$. Composite Material, 4, 20-35 (1970) 


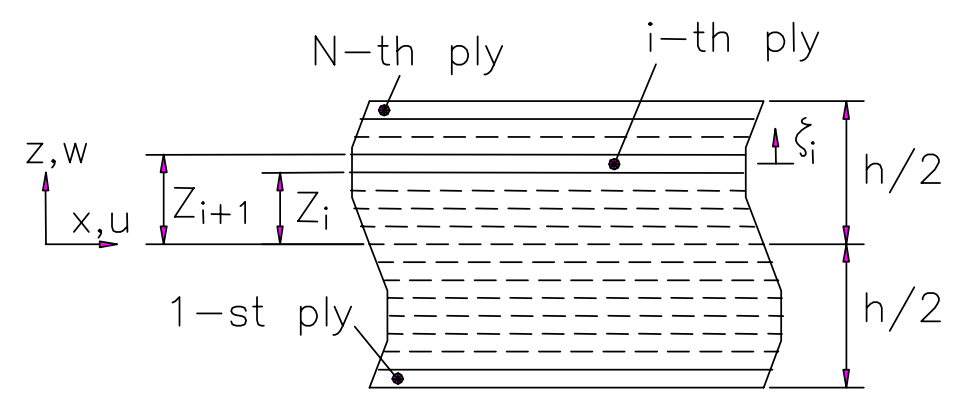

Figure 1. Cross-section of a N-ply laminated plate

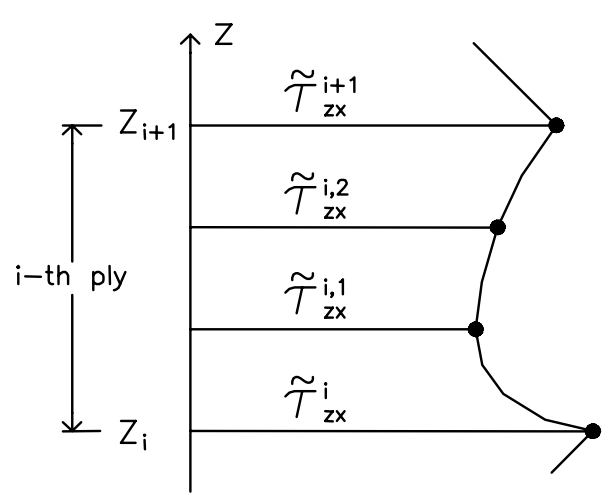

Figure 2 . The i-th ply is equally divided into three intervals for interpolating $\tilde{\tau}_{z x}$ within the ply
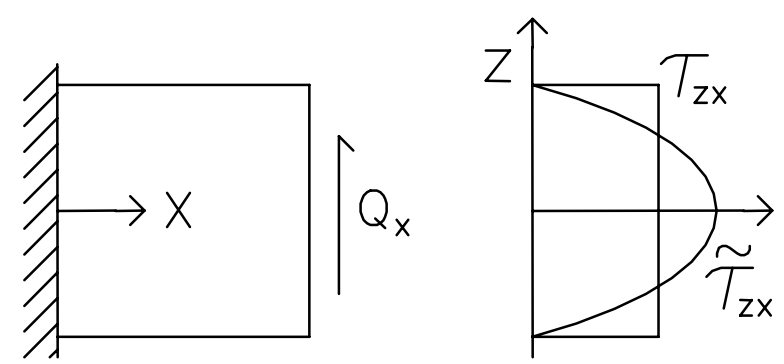

Figure 3. Transverse shear stresses $\tilde{\tau}_{z x}$ and $\tau_{z x}$ predicted respectively by theory of elasticity and the first order Mindlin theory when a shear force $Q_{x}$ is acting 


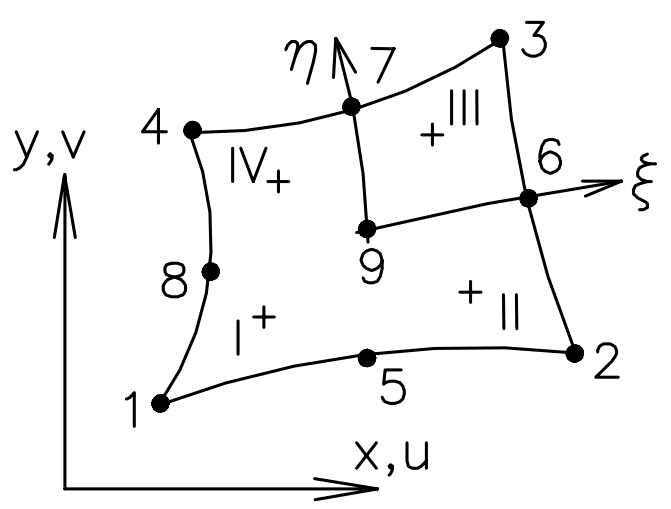

Figure 4. A nine-node quadrilateral element and its four second order Gaussian stations

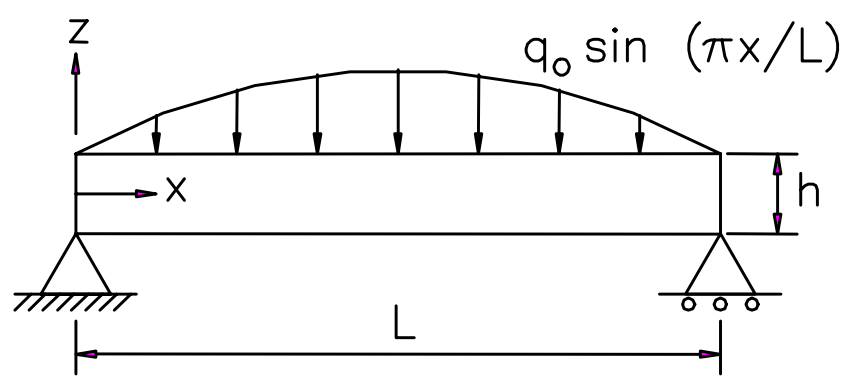

Figure 5. Simply supported laminated plate subjected to sinusoidal distributed force

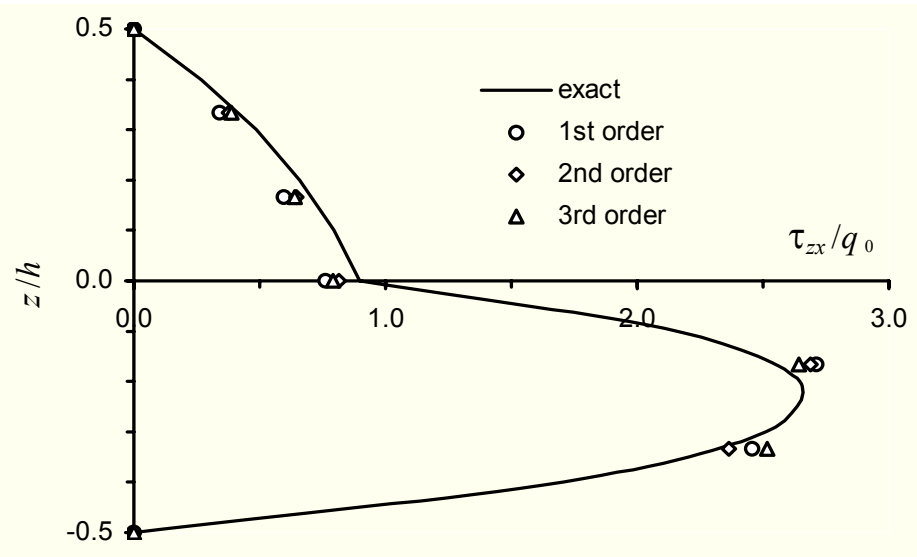

Figure 6. Thickness variation of the transverse shear stress for the two-ply plate with $S=4$ under cylindrical bending 


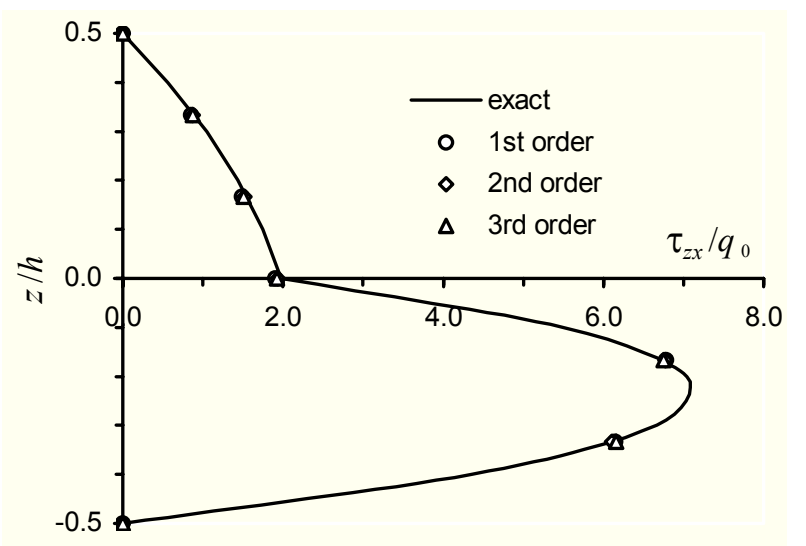

Figure 7. Thickness variation of the transverse shear stress for the two-ply plate with $S=10$ under cylindrical bending

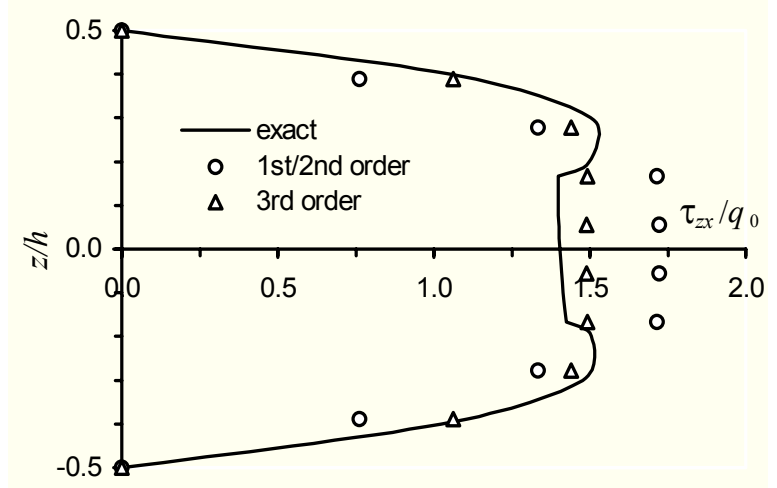

Figure 8. Thickness variation of the transverse shear stress for the three-ply plate with $S=4$ under cylindrical bending

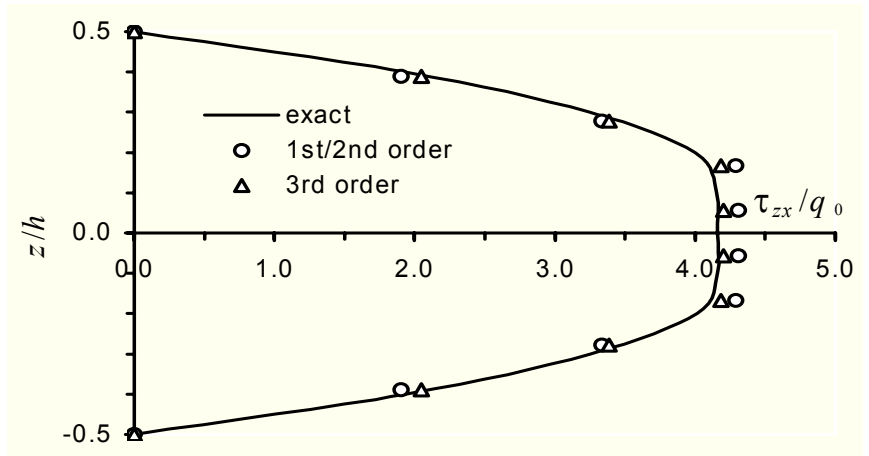

Figure 9. Thickness variation of the transverse shear stress for the three-ply plate with $S=10$ under cylindrical bending 


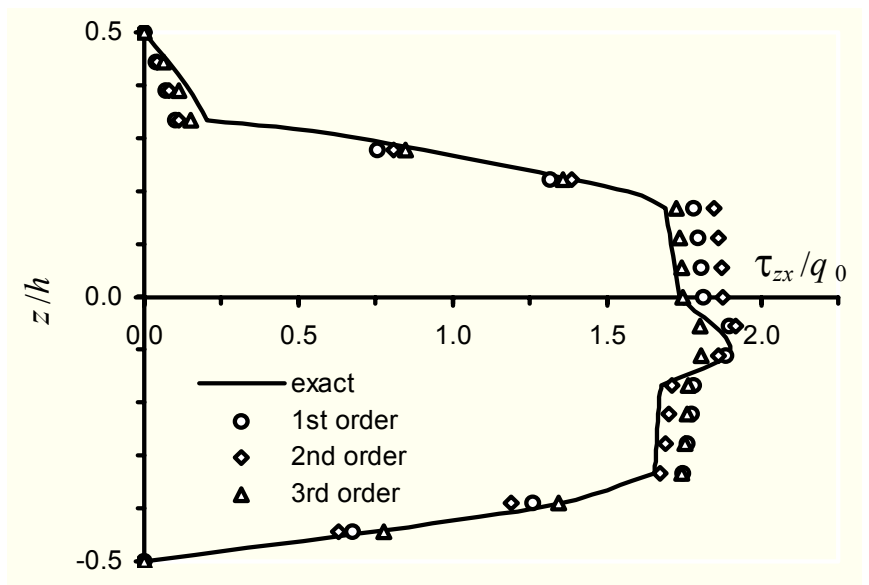

Figure 10. Thickness variation of the transverse shear stress for the six-ply plate with $S=4$ under cylindrical bending

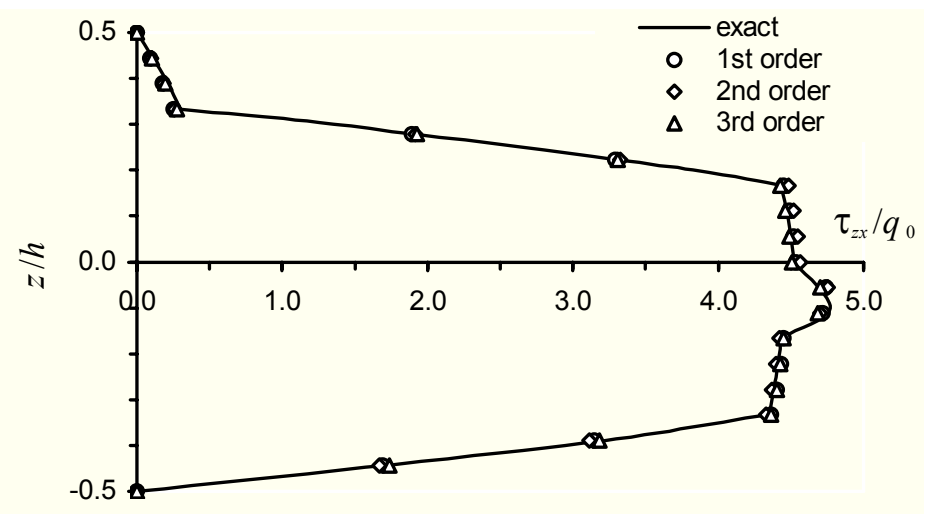

Figure 11. Thickness variation of the transverse shear stress for the six-ply plate with $S=10$ under cylindrical bending

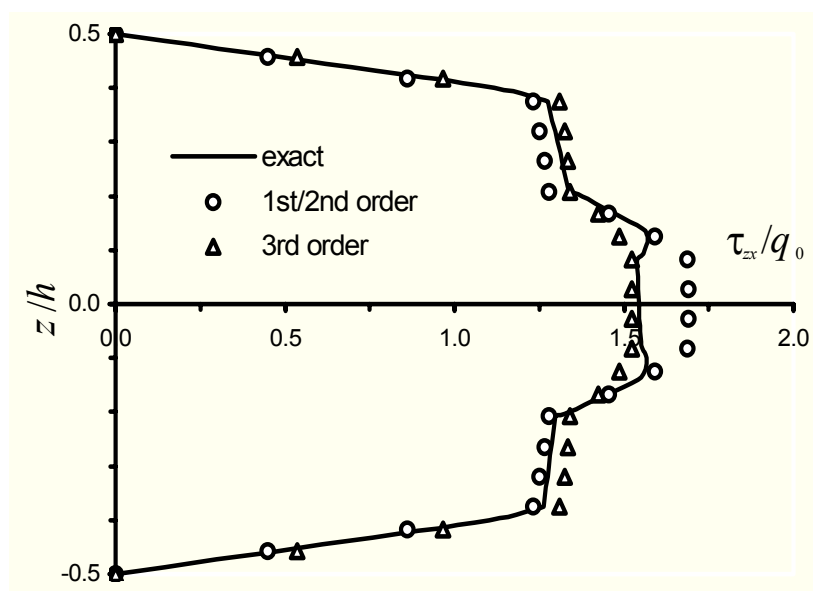

Figure 12. Thickness variation of the transverse shear stress for the seven-ply plate with $S=4$ under cylindrical bending 


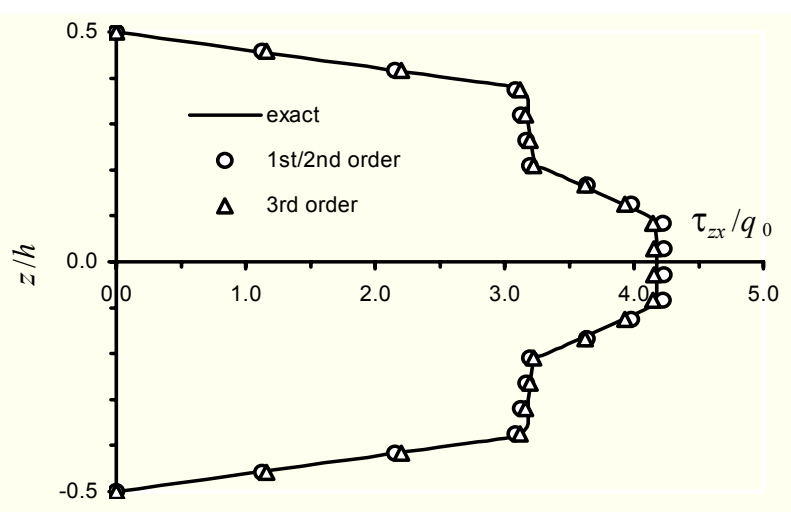

Figure 13. Thickness variation of the transverse shear stress for the seven-ply plate with $S=10$ under cylindrical bending

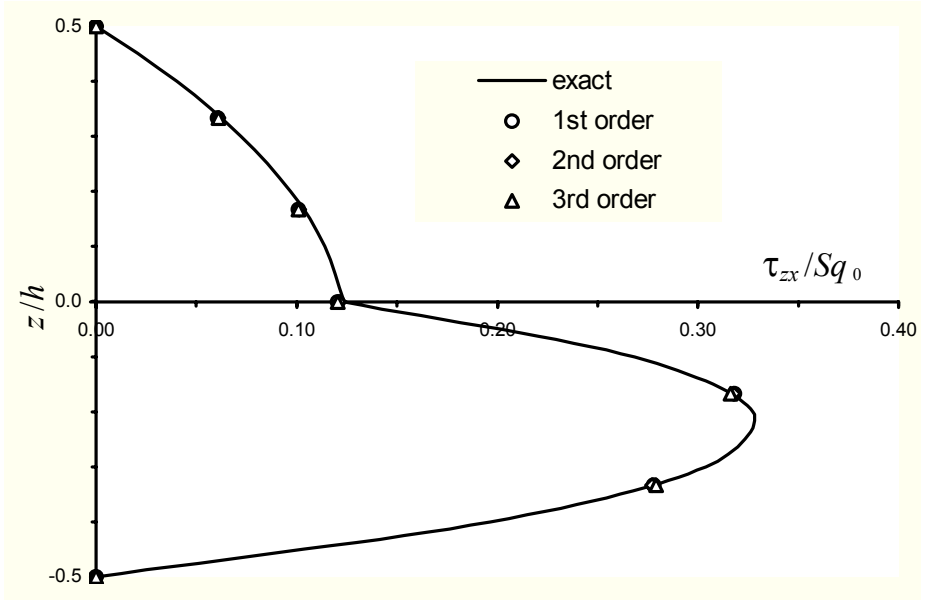

Figure 14. Thickness variation of the transverse shear stress $\tau_{z x}$ for the 2-ply square plate with $S=10$ at $x=l / 32, y=15 l / 32$

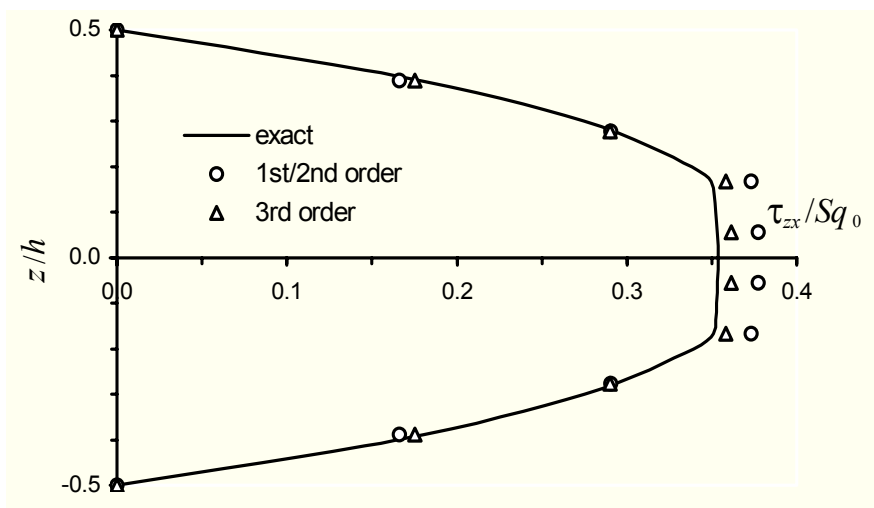

Figure 15. Thickness variation of the transverse shear stress $\tau_{z x}$ for the 3-ply square plate with $S=10$ at $x=l / 32, y=15 l / 32$ 


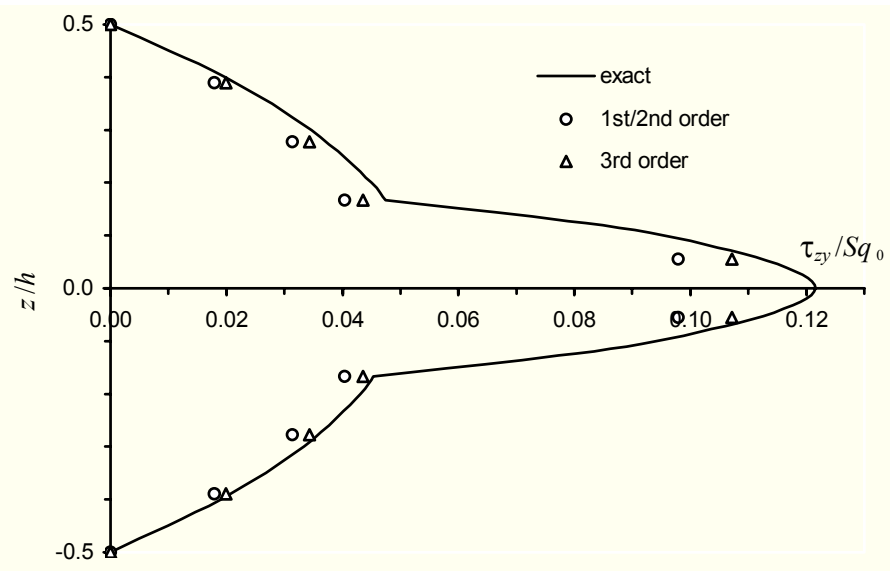

Figure 16. Thickness variation of the transverse shear stress $\tau_{z y}$ for the 3-ply square plate with $S=10$ at $x=15 l / 32, y=l / 32$

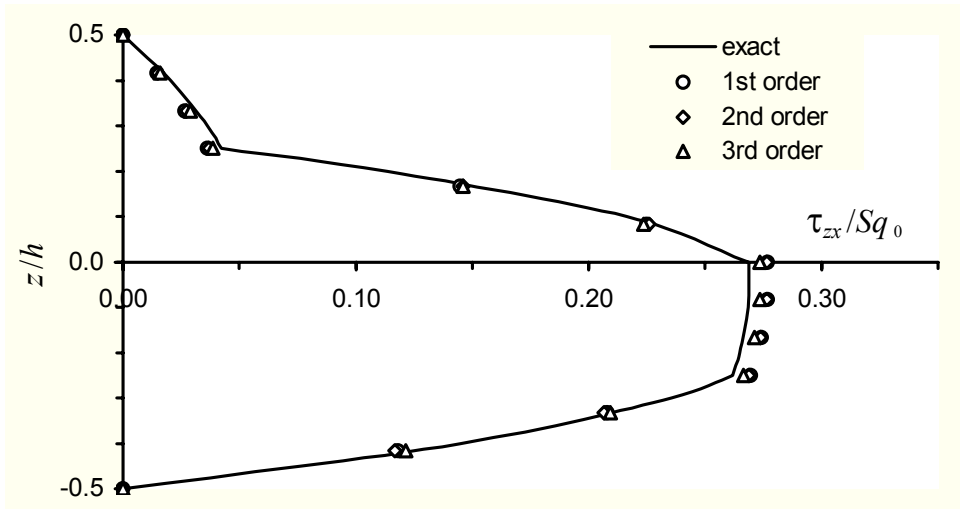

Figure 17. Thickness variation of the transverse shear stress $\tau_{z x}$ for the 4-ply square plate with $S=10$ at $x=l / 32, y=15 l / 32$

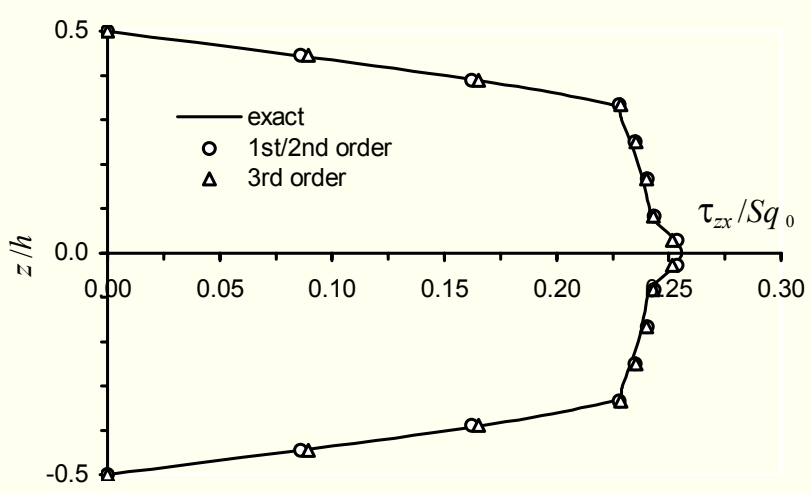

Figure 18. Thickness variation of the transverse shear stress $\tau_{z x}$ for the 5-ply square plate with $S=10$ at $x=l / 32, y=15 l / 32$ 


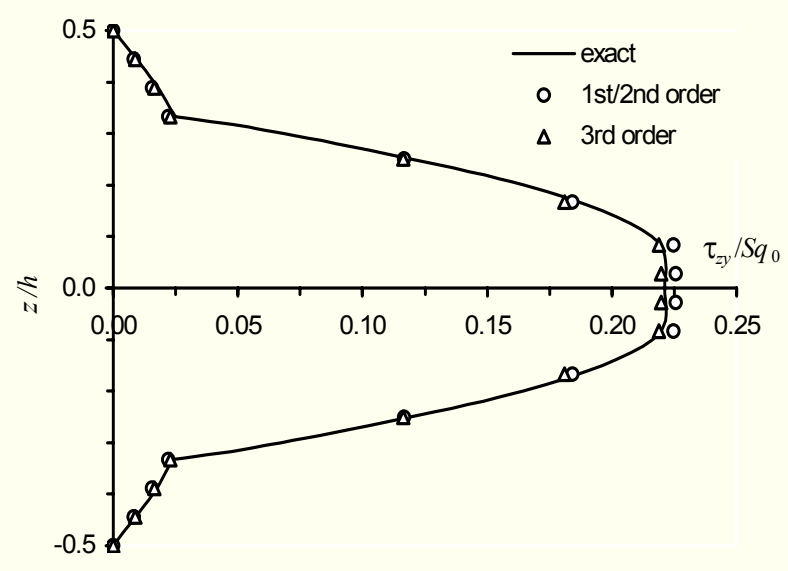

Figure 19. Thickness variation of the transverse shear stress $\tau_{z y}$ for the 5-ply square plate with $S=10$ at $x=15 l / 32, y=l / 32$

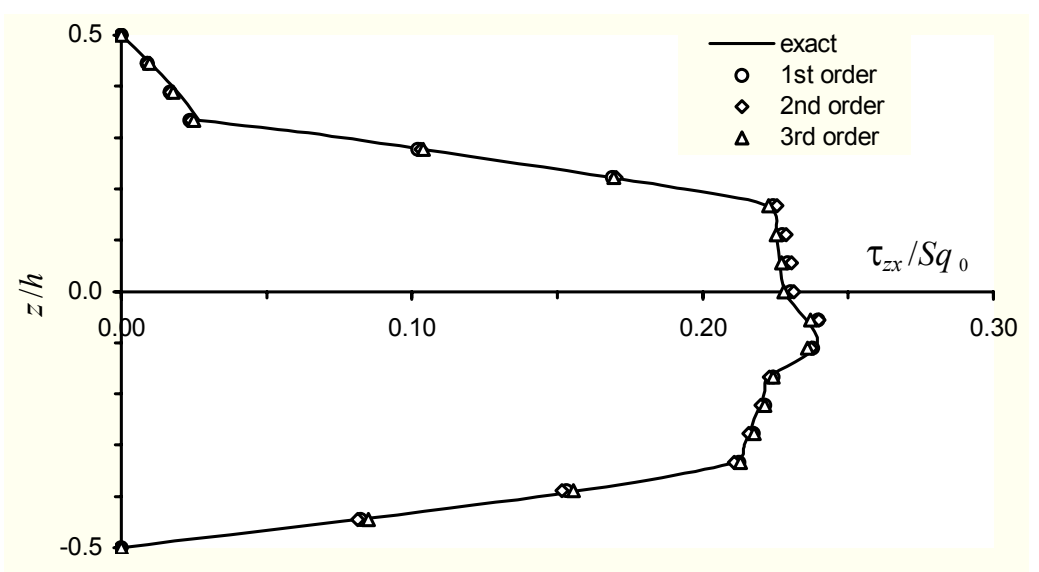

Figure 20. Thickness variation of the transverse shear stress $\tau_{z x}$ for the 6-ply square plate with $S=10$ at $x=l / 32, y=15 l / 32$

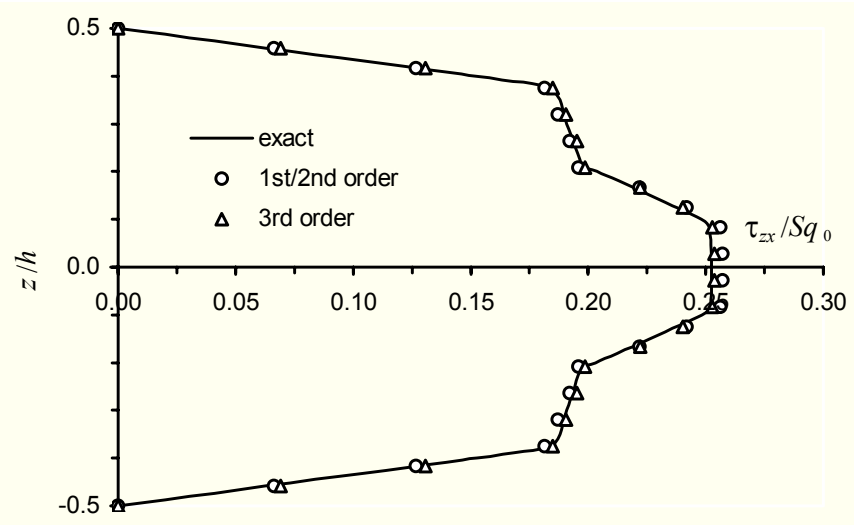

Figure 21. Thickness variation of the transverse shear stress $\tau_{z x}$ for the 7-ply square plate with $S=10$ at $x=l / 32, y=15 l / 32$ 


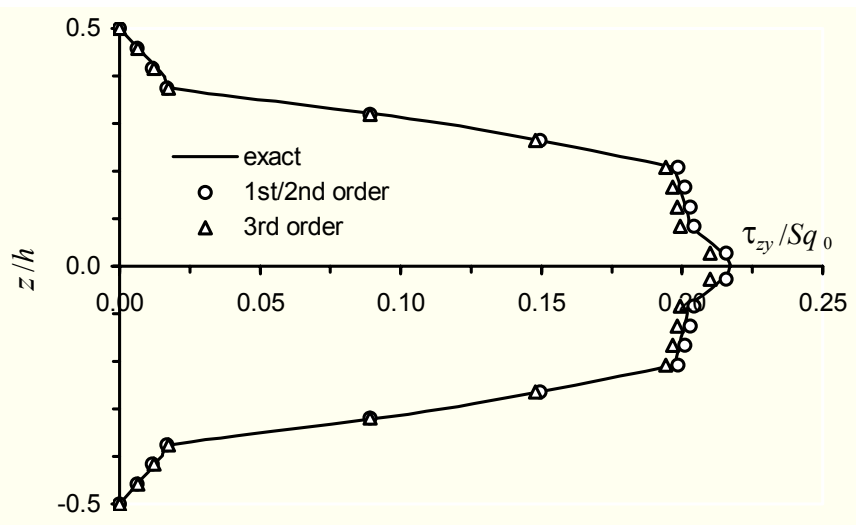

Figure 22. Thickness variation of the transverse shear stress $\tau_{z y}$ for the 7-ply square plate with $S=10$ at $x=15 l / 32, y=l / 32$

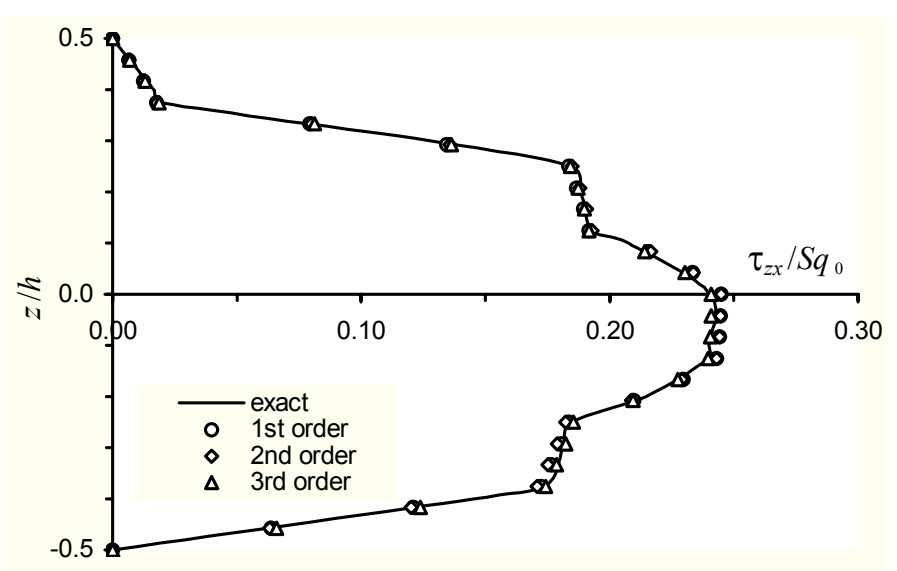

Figure 23. Thickness variation of the transverse shear stress $\tau_{z x}$ for the 8-ply square plate with $S=10$ at $x=l / 32, y=15 l / 32$

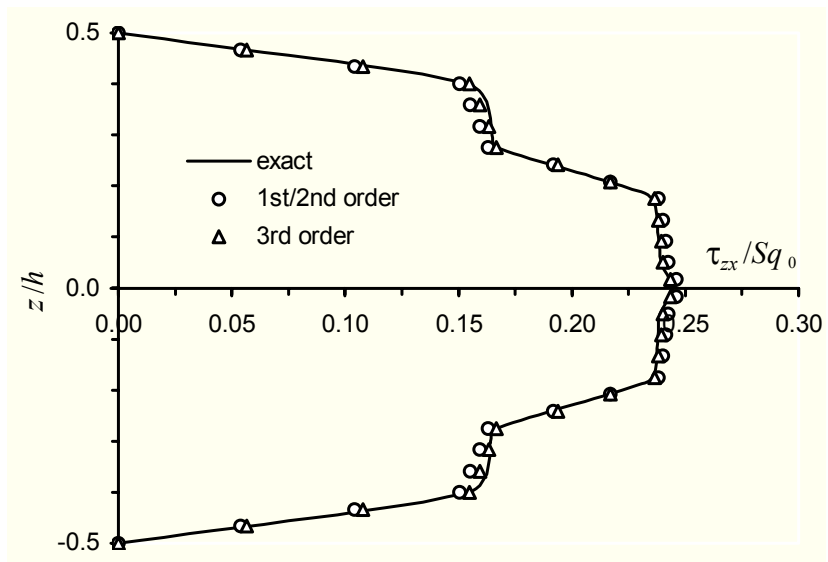

Figure 24. Thickness variation of the transverse shear stress $\tau_{z x}$ for the 9-ply square plate with $S=10$ at $x=l / 32, y=15 l / 32$ 


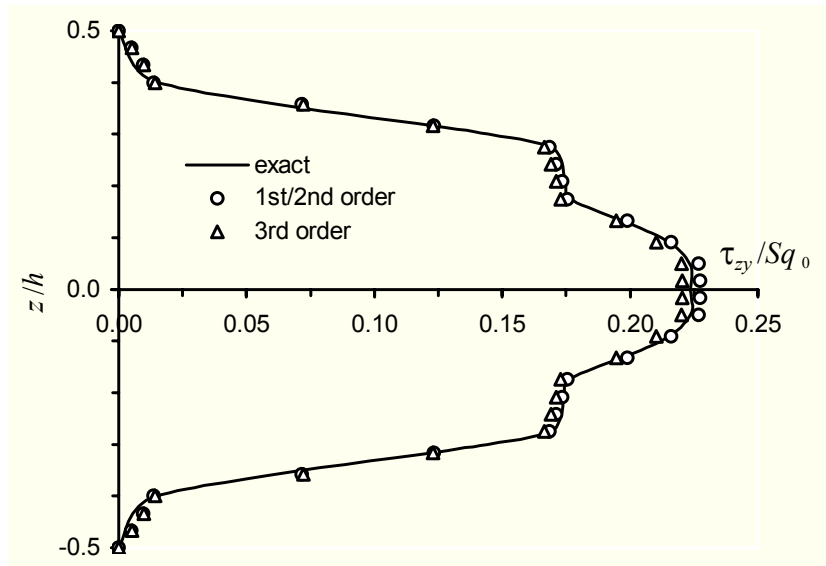

Figure 25. Thickness variation of the transverse shear stress $\tau_{z y}$ for the 9-ply square plate with $S=10$ at $x=15 l / 32, y=l / 32$

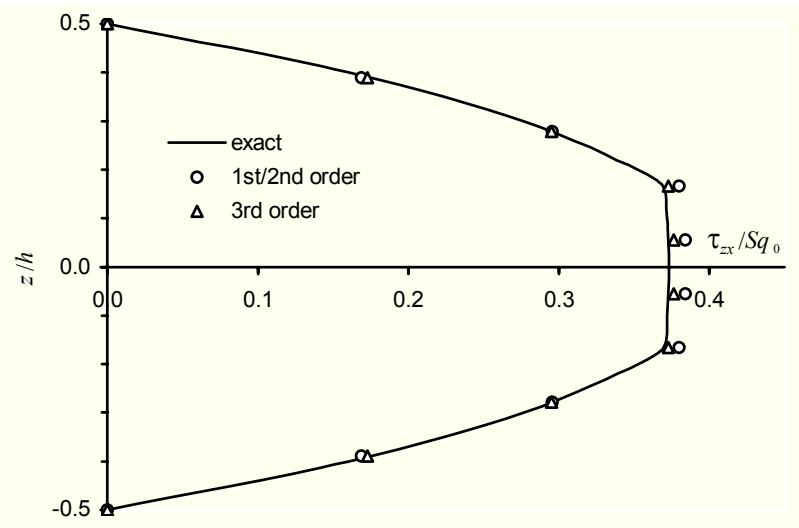

Figure 26. Thickness variation of the transverse shear stress $\tau_{z x}$ for the 3-ply square plate with $S=15$ at $x=l / 32, y=15 l / 32$

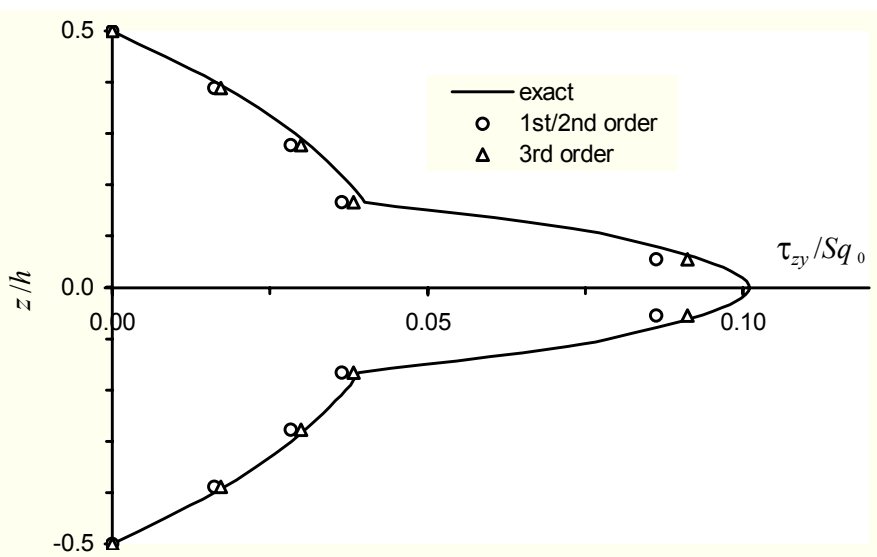

Figure 27. Thickness variation of the transverse shear stress $\tau_{z y}$ for the 3-ply square plate with $S=15$ at $x=15 l / 32, y=l / 32$ 